\title{
Frontières
}

\section{Qui est le fou ?}

\section{Entre raison et déraison}

\section{Martyne-Isabel Forest}

Volume 13, numéro 2, printemps 2001

Les morts de l'esprit

URI : https://id.erudit.org/iderudit/1074459ar

DOI : https://doi.org/10.7202/1074459ar

Aller au sommaire du numéro

\section{Éditeur(s)}

Université du Québec à Montréal

ISSN

1180-3479 (imprimé)

1916-0976 (numérique)

Découvrir la revue

Citer ce document

Forest, M.-I. (2001). Qui est le fou ? Entre raison et déraison. Frontières, 13(2), 58-66. https://doi.org/10.7202/1074459ar

\section{Résumé de l'article}

Qui est le fou ? Un être dont l'opinion est par nature déraisonnable, incapable de consentir ou de refuser un traitement ? Sa condition le rend-il inapte à déterminer ce qui constitue son bien ? Dans quelle mesure sommes-nous prêts et capables de lui permettre d'exercer ses droits à l'autonomie et à

l'autodétermination, en tant que juristes, en tant que psychiatres ? Selon notre hypothèse, les acteurs du procès portant sur l'autorisation de traiter le fou contre son gré seraient fortement imprégnés des représentations sociales de la folie. Victime de ce regard-jugement, le fou serait soumis au pouvoir des experts, de l'argument de la santé qu'ils promeuvent et de leur influence chez les décideurs. Nous plaidons en faveur d'une liberté recouvrée pour ces personnes trop souvent captives.
Ce document est protégé par la loi sur le droit d'auteur. L'utilisation des services d'Érudit (y compris la reproduction) est assujettie à sa politique d'utilisation que vous pouvez consulter en ligne.

https://apropos.erudit.org/fr/usagers/politique-dutilisation/ 
Résumé

Qui est le fou ? Un être dont l'opinion est par nature déraisonnable, incapable de consentir ou de refuser un traitement? Sa condition le rend-il inapte à déterminer ce qui constitue son bien? Dans quelle mesure sommes-nous prêts et capables de lui permettre d'exercer ses droits à l'autonomie et à l'autodétermination, en tant que juristes, en tant que psychiatres? Selon notre hypothèse, les acteurs du procès portant sur l'autorisation de traiter le fou contre son gré seraient fortement imprégnés des représentations sociales de la folie. Victime de ce regardjugement, le fou serait soumis au pouvoir des experts, de l'argument de la santé qu'ils promeuvent et de leur influence chez les décideurs. Nous plaidons en faveur d'une liberté recouvrée pour ces personnes trop souvent captives.

Mots clés: fou - inaptitude refus de traitement-procèsreprésentations sociales

\section{Abstract}

Who is called the fool? Is he someone who's opinion is automatically viewed as nonsensical, being unable to consent or to refuse a treatment? Does his condition induce an inaptitude to determine what is right for him ? Are we ready and capable, as jurists and as psychiatrists, to help the fool earn the right to selfdetermination, to autonomy? According to our hypothetical view on this issue, the protagonists of the legal processes would be profoundly influenced by the social representations of madness. Victim of such prejudices, the fool is compelled to submit himself to the power of the health experts, on their strong influence on the decision-makers. We are claiming more freedom for those who are, all too often, prisoners of our narrow-minded conception of their disease.

Key words: fool-incapacity-treatment refusal-trial-social representations

\section{Qui est le fou? Entre raison et déraison}

MOURIR SA VIE, MOURIR LE TEMPS, MOURIR LA PEUR, MOURIR LE NÉANT. LA MORT, J'AVAIS EXAMINÉ LA QUESTION DE PRÈS: LA MORT C'ÉTAIT LE PLAFOND. QUAND ON CONNAÎT LE PLAFOND MIEUX QUE SOI-MÊME, CELA S'APPELLE LA MORT. LE PLAFOND EST CE QUI EMPÊCHE LES YEUX DE MONTER ET LA PENSÉE DE S'ÉLEVER. QUI DIT PLAFOND DIT CAVEAU: LE PLAFOND EST LE COUVERCLE DU CERVEAU.

A. NOTHUMB, LA MÉTAPHYSIQUE DES TUBES, 2000, p. 54

\section{Me Martyne-Isabel Forest, LL.M., consultante en droit des personnes et en humanisation des soins, \\ Direction générale, Hôpitaux universitaires de Genève; responsable du programme et de la formation, Certificat de formation continue en éthique clinique, Centre interfacultaire de gérontologie, Université de Genève, Suisse.}

Un sujet grave. Vous l'aurez pressenti, c'est de l'enfermement du fou, dans son sens le plus large, dont il s'agit ici de parler et du processus qui l'y entraîne.

Des sujets graves aussi : la préservation du soi, dont on ne fait l'expérience qu'à la condition d'être "sujet de son action, centre de sa vie ${ }^{1}{ }^{»}$ et de l'intégrité physique et psychique des personnes, introduits d'abord en empruntant les mots d'Amélie Nothumb dans La métaphysique des tubes, pour saisir l'extraordinaire cruauté de l'isolement dans lequel on confine la personne dont on dit qu'elle déraisonne, le plus souvent contre son gré mais avec l'autorisation du prétoire, selon la procédure prévue, l'ancien décret de prise du corps, à des fins thérapeutiques, bien entendu....

N'est-ce pas là le seul moyen que l'on prescrive encore dans nos sociétés dites civilisées pour protéger le dément contre lui-même ou pour protéger la collectivité : l'encadrer de murs et d'un plafond justement, le séparer de l'Autre, le mettre à distance, au rancart, dans des institutions spécialisées, entouré de professionnels spécialisés, en le privant du regard et là, peut-être, « le mettre à mort ». Le regard que l'on porte sur celui dont l'esprit divague est si mortifère.

Or, la vie, ou plutôt ici la mort, commence là où commence le regard, comme la parole du reste:

Les yeux des êtres vivants possèdent la plus étonnante des propriétés : 
le regard. Il n'y a pas plus singulier. Qu'est-ce que le regard ? C'est inexprimable. Aucun mot ne peut approcher son essence étrange. Et pourtant le regard existe. Il y a même peu de réalités qui existent à ce point.

Quelle est la différence entre les yeux qui ont un regard et les yeux qui n'en ont pas? Cette différence a un nom: c'est la vie... ${ }^{2}$

Aussi, il s'agira dans un premier temps de définir le fou et de déterminer sa capacité sous les regards du droit et de la clinique pour en dégager, dans un deuxième temps, les impacts dans l'action, c'est-à-dire au moment même du procès qu'il subit pour avoir refusé un traitement. Avec le verbe parfois cinglant, nous aborderons alors la décision judiciaire d'administrer un traitement contre le gré d'une personne atteinte de maladie mentale à travers trois scénarios : le procès a lieu, le fou en est-il informé, est-il présent?; le procès a lieu, le fou est présent, l'entendrons-nous?; et, enfin, le procès a lieu, le fou est présent, mais le témoin-expert est aussi au rendez-vous... ${ }^{3}$

À l'occasion du traitement de ces sujets, nous ouvrirons une brèche dans différentes certitudes dont celle du procès conçu trop sommairement comme « un litige soumis à un tribunal »; celle de l'art de juger, comme le résultat d'une décision purement rationnelle prise par un être impartial, indifférent aux représentations de la folie; celle du concept de maladie mentale, comme étant objectif, en tout cas clair et juste.

Autant de certitudes qui ne permettent pas d'appréhender ces phénomènes dans leur fabuleuse complexité : «la certitude ne tolère, ne leur faisant nulle place, ni le doute, ni la différence, ni la divergence pas même la nuance ${ }^{4}$ ». Par là, nous évoquons aussi la possibilité que les protagonistes dans cette affaire construisent collectivement la connaissance et la décision qu'ils sont appelés à prendre comme un travail de réduction d'une situation d'incertitude. On sait que c'est le cas de situations où sont mis en présence des intérêts divergents relativement à l'action à prendre. L'exemple pratique de la décision d'administrer un traitement contre le gré d'une personne atteinte de maladie mentale forme à ce titre un objet d'analyse de choix!

Enfin, disons-le, notre réflexion a comme toile de fond notre propre conscience juridique, sociale, morale, à laquelle nous admettons d'emblée ne pouvoir échapper. Nous adhérons à l'idée que la conscience juridique de la classe sociale dominante dans une société donnée se reflète dans le droit en vigueur. En son centre, cet article est donc aussi traversé par nos sentiments face à la justice, au pouvoir: "Tu es celui qui écrit et qui est écrit », a dit Jabès... ${ }^{5}$

\section{QUI EST LE FOU ET EST-IL APTE À DÉCIDER DE SON PROPRE BIEN ?}

Comment peut-on entendre et considérer la voix d'un simple d'esprit, d'un aliéné, d'un insensé, d'un irresponsable, d'un forcené, d'un halluciné, d'un inconscient, d'un possédé, d'un déséquilibré, d'un être maléfique? Un corps privé de raison. $\mathrm{Vu}$ sous cet angle, difficile de résister à la tentation de l'enfermer en effet! Et, c'est vrai par ailleurs qu'il faille le faire dans certains cas précis. Mais encore, qui est le fou?

À la suite de Bobin, pour éviter tout malentendu, nous avons envie de dire: «Je sais bien qu'on ne doit pas dire fou mais handicapé mental ou quelque chose comme ça. Mais je préfère le mot fou. Il est plus rapide et il sonne comme doux ${ }^{6} »$. Presque toujours, nous utiliserons ici le mot «fou», à dessein, sans doute parce nous avons ressenti qu'il s'agissait là de la meilleure façon de mettre en scène l'injustice dont il peut être victime en raison de son état de santé. De toute manière, un seul coup d'œil sur son expérience de l'hospitalisation psychiatrique suffit à un être normalement sensible pour ne pas douter que le fou puisse effectivement être considéré comme une victime de notre fonctionnement social et du type de solutions que nous proposons. Voici, pour décrire ce qui se passe, une énumération étonnante, inquiétante, élaborée à partir des patients eux-mêmes, de leurs savoirs propres, que nous vous proposons de lire et d'entendre sans les envisager surtout - comme des savoirs lacunaires «susceptibles d'être redressés, complétés, corrigés par un savoir médical conforme ${ }^{7} »$ :

- la domination par le personnel et l'infantilisation dont les sujets se sentent victimes;

- l'emploi systématique des médicaments psychotropes, qui prennent alors la place d'une écoute et d'une aide réelles; leurs effets secondaires débilitants ;

- la quotidienneté marquée par l'ennui et les règlements sévères, le fonctionnement institutionnel par récompenses-punitions;

- l'absence d'aide réelle permettant au sujet de sortir vraiment des difficultés qui l'ont mené en psychiatrie ;

- l'obligation dans laquelle le rapport chronique à l'institution met les sujets de faire leur deuil d'une vie normale et investie ;

- la perte des droits instituée par la psychiatrisation, l'arbitraire de l'hospitalisation obligatoire;

- l'intrusion dans la vie intérieure, personnelle et intime du sujet que représente l'intervention psychiatrique;
- la réinsertion sociale pavée de difficultés, l'invalidation marquée et la solitude que le passage en psychiatrie entraîne ${ }^{8}$. Pour définir le fou, revenons au maîtrelivre de Foucault, qui le décrit «comme étant cet individu dangereux qui, à l'inverse $\mathrm{du}$ délinquant qui n'en viole qu'une, peut violer toutes les règles »; "une transgression vivante dont on cherche à se protéger $»^{9}$. En vérité, on en a tellement peur: «Pas besoin de gril, disait Sartre, l'enfer, c'est les Autres. » Historiquement, toutes les sociétés se sont hardiment engagées à s'en protéger. C'est là qu'à notre sens une réflexion critique sur l'influence du regardjugement, du regard-verdict, prend toute sa pertinence. Lacan n'a-t-il pas dit: «C'est le regard de l'autre qui me construit»?

En tout cas, à l'instar de nous tous, le fou est un «il » parlé par les autres, un autre à soi-même :

Le Je du sujet parlant, son identité donc, ou plus exactement ce à partir de quoi il croit pouvoir dire Je, est constitutivement aliéné.

Car le Je s'identifie non pas à partir

d'une intériorité, mais à partir d'un reflet spéculaire qui lui est renvoyé dans le regard porté sur lui. Ce regard est comme un sceau qui ne cesse de s'imprimer en lui, et auquel il n'échappera plus ${ }^{10}$.

Chaumon pose le problème de la folie en des termes semblables: "La folie c'est ce qui est repéré par autrui comme excès insupportable alors même que celui qui en est la proie ne la nomme pas ainsi ${ }^{11} »$. Or selon lui, seuls les mots du fou peuvent nous permettre d'accéder à ce qu'il est. Il n'est pas question de s'en remettre à ce qu'il est dit sur lui. N'avons-nous pas, pour définir le fou autrement, pour devenir plus responsables, le «devoir de penser », comme l'écrit Hannah Arendt dans son Rapport sur la banalité du mal ${ }^{12}$ :

Mais accomplir notre "devoir

de penser », " obéir créativement»

est une entreprise difficile et périlleuse car cela implique en quelque sorte que nous bravions l'interdit de penser aussi arbitraire qu'implicite que véhicule l'idéologie scientiste lorsqu'elle rejette comme dépourvues de sens les questions qui ne sont pas susceptibles de recevoir une réponse technique ${ }^{13}$.

\section{DANS LES UNIVERS CLINIQUE ET JURIDIQUE, LE FOU ÉCHAPPERA-T-IL AU REGARD «FOLIE-MALADIE »?}

Pour vraiment être en mesure d'évaluer l'impact de nos préjugés, de nos préconceptions, de nos regards et de nos peurs du fou dans les univers de la clinique et du droit, nous estimons devoir au moins évoquer, même partiellement, ce que l'on appelle 
les représentations sociales de la folie. Il faudrait absolument en effet conserver à l'esprit qu'elles «orientent au présent, nos savoirs, nos opinions, nos jugements ainsi que nos pratiques » et qu'elles «s'infiltrent au cour des discours tenus par les spécialistes eux-mêmes ${ }^{14}$. Pas banal du tout. Un passage qu'il faut peut-être relire.

D'après la recherche menée par Schurmans, bien qu'il existe des espaces de sens basés sur des modèles psychosociaux qui considèrent la personne folle de façon beaucoup plus englobante, c'est encore malheureusement le modèle biomédical qui prédomine au niveau des représentations sociales de la folie:

Les résultats indiquent combien la définition médicale de la foliemaladie s'impose et se diffuse dans les milieux non spécialisés, dès le XVIII ${ }^{\mathrm{e}}$ siècle. Cette conception se dote d'un appareillage fort

- les établissements hospitaliers, les programmes de formation médicale, la précision accrue des catégories nosographiques, les textes de loi en témoignent - qui la constitue en convention dominante ${ }^{15}$.

Notre interrogation, à la fois fondamentale et détestable aux yeux de certains : par quel miracle le droit, dans sa rencontre avec la médecine sur l'étrangeté de la folie, échapperait-il à l'emprise de ce modèle biomédical? Une hypothèse nécessairement délirante ou choquante?!

La déférence généralement témoignée envers le psychiatre, les représentations sociales de la folie dont le juge subirait également l'influence selon notre hypothèse, le recours à l'expertise psychiatrique fondée sur une reconnaissance du savoir spécialisé, nécessaire au niveau de la prise de décision bien qu'accompagné il est vrai d'une plus ou moins grande adhésion par les juges, le fait que les professionnels du droit se savent et se disent non spécialisés et, enfin, l'absence d'un véritable débat contradictoire, paraissent restreindre considérablement la liberté de jugement et de décision de toutes les personnes présentes au procès. Or, s'ils sont plus ou moins libres dans la formation de leur regard-jugement, que dire de l'espace de liberté du fou?

Retournons d'abord à la petite histoire de la reconnaissance juridique de l'aptitude du fou ou, en d'autres termes, de son droit à consentir ou à refuser un traitement. Allons voir rapidement d'où l'on vient avant d'examiner ce que l'on fait aujourd'hui de ce droit, c'est-à-dire de quelle façon on l'applique dans les faits. Au moment des débats concernant la question de savoir si le fou pouvait, à l'instar des autres personnes, posséder le droit à l'autonomie, deux valeurs se sont opposées avec force: celle de la santé, défendue par les membres de la profession psychiatrique et celles de l'égalité et de l'autonomie, défendues par les maîtres de l'approche légale, attachés à la protection des droits de la personne. Lequel des deux clans l'emportera ? À cette première étape, celui qui promeut les droits de la personne. Mais la guerre ne s'arrêtera pas là.

C'est au moment d'appliquer le droit, dans l'agir, quand on quitte le moment des grands discours, que le clan psychiatrique reviendra en force et s'imposera, notamment en raison d'un argument massue, qui ne laisse personne indifférent, celui de l'efficacité thérapeutique. Force est de constater que devant l'autorité d'un tel argument, l'on doive s'incliner assez bas. On verra l'argument de la santé finir par l'emporter haut la main et les discours, mordre la poussière.

\section{LA PETITE HISTOIRE DE LA RECONNAISSANCE DE LA CAPACITÉ JURIDIQUE DU FOU}

À l'avant-scène du jeu de l'élaboration des textes de lois et des principes qui couvrent le champ de la santé mentale, les rôles principaux sont incontestablement tenus par les acteurs du droit et de la psychiatrie, qui sont le plus souvent des alliés du reste. Mais ici, la question du consentement au traitement fera apparaître leurs principales divergences, formera le lieu de leur opposition: "Le conflit émerge plus particulièrement lorsque l'ordre juridique cherche à encadrer le pouvoir des psychiatres à l'égard des patients dans la décision de traitement ${ }^{16}$.»

Le conflit entre l'ordre psychiatrique et l'ordre juridique se cristallise autour de deux valeurs ${ }^{17}$ : la santé contre l'égalité et l'autonomie. Essentiellement binaire, la certitude se fera ici guerrière.

\section{L'argument de la santé comme valeur}

Pour le clan psychiatrique, il est fondamental de pouvoir admettre une personne en psychiatrie et/ou de pouvoir la traiter, sans nécessairement obtenir son consentement, afin de s'assurer qu'elle bénéficie effectivement des soins que sa condition requiert, cette dernière étant à l'origine, comme en témoignent les experts psychiatriques de l'OMS, de son incapacité de comprendre qu'elle a besoin d'être hospitalisée et traitée ${ }^{18}$.

Le fou doit avoir accès aux meilleurs services de santé, au même titre que toutes les autres catégories de patients et, surtout, sans entrave de la part de la loi ou encore avec son autorisation ou sa protection, cette décision étant de nature strictement médicale :

La tradition médicale considère toutes les questions relatives au traitement comme étant des questions de nature médicale, y compris la décision d'accepter ou non de se soumettre à un traitement. Traditionnellement aussi, l'ordre juridique n'échappe pas à cette conception paternaliste quand il reconnaît ce pouvoir au psychiatre ${ }^{19}$.

Pourquoi diable se refuser à l'évidence? ! On se doit de protéger le droit à la santé du fou, du révolté qui refuserait sa condition, envers et contre tous, sans discrimination. L'argument de la santé comme valeur, irréfutable, ferait résolument contrepoids aux valeurs de liberté, d'égalité et d'autonomie car «si l'accent est mis sur la protection du droit du patient à la liberté dans de telles circonstances, c'est inévitablement aux dépens de son droit aux soins et aux traitements. Le droit de refuser un traitement est un empêchement à la réalisation du droit au traitement et, par conséquent, du droit à la santé20». Que répondre à cela? Le respect du droit à la santé est tellement fondamental.

Voici la conclusion presque inquiétante à laquelle en vient Gendreau à la suite de son analyse des valeurs qui fondent le discours des professionnels psychiatriques:

[...] la loi doit servir d'abord et avant tout à faciliter leur travail dans l'intérêt des malades mentaux. Pour ce faire, elle doit consacrer leur autonomie professionnelle, elle doit autoriser leur intervention auprès des patients non consentants qui ont besoin de traitements ${ }^{21}$

\section{L'argument de l'égalité}

et de l'autonomie comme valeurs

D'abord LA question: pourquoi toute personne malade physiquement aurait-elle le droit de refuser un traitement quand bien même cela entraîne pour sa santé des conséquences très négatives, voire la mort dans certains cas, et que cela ne serait pas possible pour un patient psychique ? On voit poindre la valeur égalité dans ce discours, en même temps que le droit à la différence.

$\mathrm{Au}$ cours de l'élaboration des principes de l'ONU concernant cette question, l'Organisation internationale des personnes handicapées (OIPH) soutiendra que «les patients sont souvent les mieux placés pour évaluer les bienfaits d'un traitement, en particulier lorsqu'il s'agit de neuroleptiques », problème épineux s'il en est dans le contexte du refus de traitement en psychiatrie ${ }^{22}$.

Le Comité d'experts alors réuni fait valoir que le patient admis involontairement a besoin d'une protection accrue non seulement parce qu'il est privé de sa liberté mais également parce qu'il a besoin d'être véritablement entendu, l'expression de son expérience constituant l'une des clés majeures dans l'évaluation du succès de l'intervention médicale. 


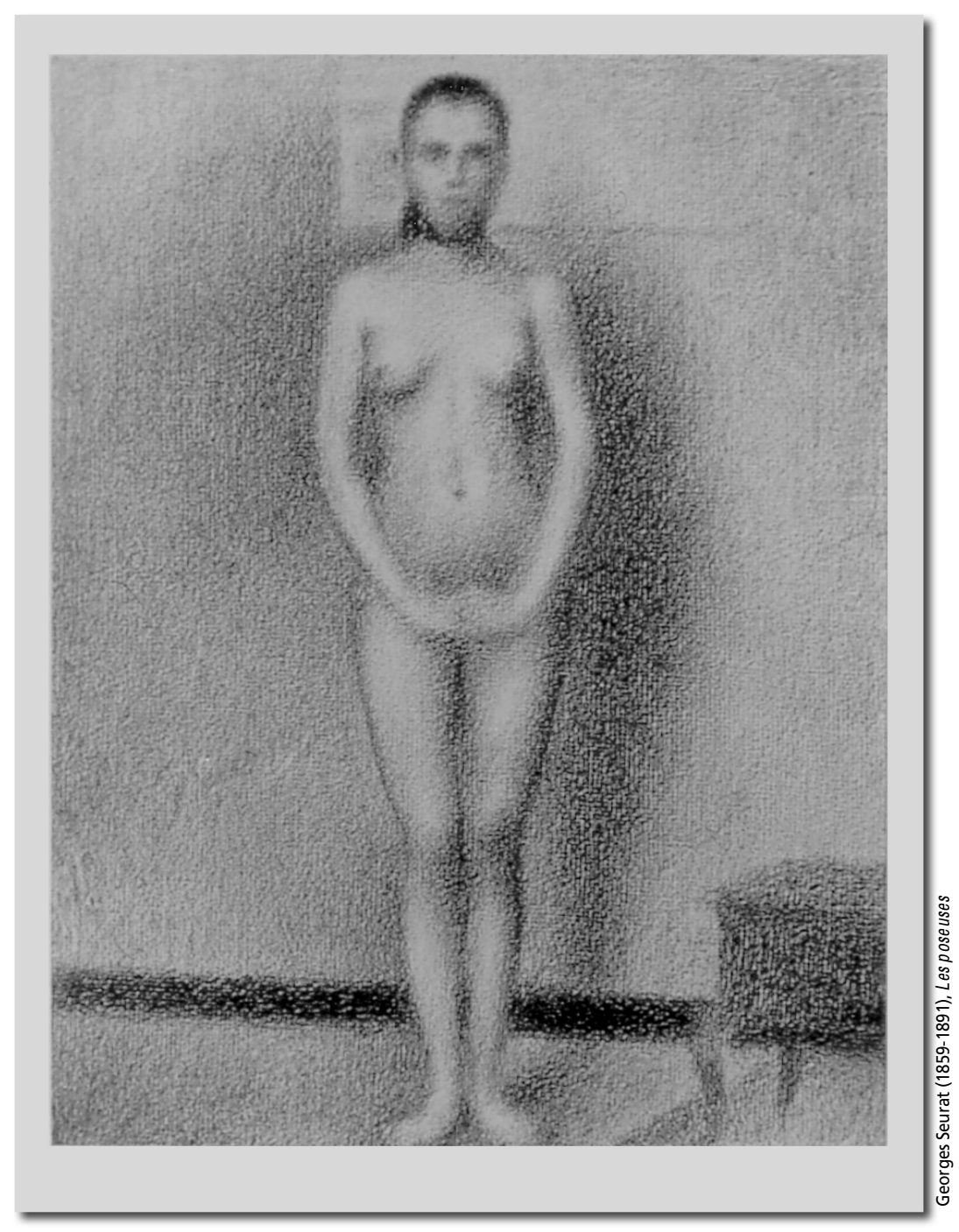

En fait, ce qui divise profondément ces deux clans tourne autour de la question de savoir si la décision d'accepter ou de refuser un traitement est ou non une décision de nature médicale? Pour les uns, comme nous l'avons déjà vu, seul l'expert psychiatre peut se qualifier. Aussi, on ne peut reconnaître au patient le droit de consentir ou de refuser, la décision relevant d'une compétence qu'il ne possède pas. Pour les autres, la décision n'est pas de nature purement médicale mais aussi profane et sociale. De plus, la loi doit protéger le patient contre le pouvoir d'intervention coercitif délégué à cet expert. On ne peut permettre que la décision repose uniquement sur l'expertise, la compétence ou l'éthique du corps médical spécialisé. Ainsi, on doit reconnaître au patient le droit de consentir ou de refuser, la décision relevant aussi de compétences qu'il possède.

À la réflexion, si la question opposant ces deux clans reposait non pas sur celle de la nature de cette décision (médicale ou non) mais sur l'autonomie du sujet psychiatrique? cité de consentir à un traitement ou de le refuser ne s'apprécie pas en fonction de la situation de l'individu mais en fonction de son autonomie décisionnelle et de sa capacité de comprendre et d'apprécier ce qui est en jeu ${ }^{25}$.»

L'aptitude et l'inaptitude sont des situations de fait. Ainsi, l'état d'esprit d'un individu, fou ou non, et son aptitude à prendre des décisions le concernant peuvent fluctuer dans le temps et varier selon plusieurs facteurs: la fatigue, la médication, l'évolution de la maladie. L'article 258 du Code civil du Québec parle "d'une maladie, d'une déficience ou d'un affaiblissement dû à l'âge qui altère les facultés mentales ou l'aptitude physique à exprimer sa volonté ». Pour déterminer l'inaptitude, certains critères ont d'abord été établis dans l'Hospitals Act de la NouvelleÉcosse, pour être repris par l'Association canadienne des psychiatres et les tribunaux québécois :

1. Le patient réalise-t-il que le psychiatre l'examine pour déterminer sa capacité et comprend-il le sens de ce terme?

2. Le patient comprend-il la nature de la maladie pour laquelle on lui propose le traitement en question?

3. Le patient comprend-il la nature et le but du traitement?

4. Le patient saisit-il les risques et les avantages du traitement s'il le subit?

5. Le patient saisit-il les risques et les avantages du traitement s'il ne le subit pas ${ }^{26}$ ?

Mais cette appréciation, faut-il le dire, n'est pas purement objective. Certains prennent le risque d'avancer que le corps médical aurait tendance à juger la personne comme étant apte lorsqu'elle accepte les conseils qu'un de ses membres lui prodigue et, à l'inverse, qu'il aurait tendance à la juger inapte lorsqu'elle les questionne et surtout lorsqu'elle les refuse. On constate également que lorsque la décision ne semble pas rationnelle ou raisonnable aux yeux de l'évaluateur de l'aptitude, on aurait tendance à juger que la personne n'est pas apte. Or, il est clair que la décision ne doit pas être jugée. L'évaluation ne doit porter que sur les habiletés d'une personne à prendre une décision. Prenons le temps d'écouter attentivement à ce propos le juge Legris, sur l'esprit de la loi justement :

Pour conclure à l'inaptitude de l'intimé à donner un consentement, il faudrait, dans les présentes circonstances, consacrer un principe voulant que toute personne qui refuse des soins médicaux jugés nécessaires par la médecine est inapte à consentir. Tel n'est pas, je crois, l'esprit de l'article 11 du Code civil du Québec ${ }^{27}$. 
Dans un deuxième temps, si le fou est considéré apte, nous devrons rechercher et obtenir de sa part un consentement libre et éclairé, exercice périlleux s'il en est. Notons que ce sont précisément les droits à l'autonomie et à l'autodétermination, à l'intégrité, à l'inviolabilité et à la dignité de la personne qui constituent les fondements juridiques et éthiques de cette obligation professionnelle, formant en conséquence son principal enjeu ${ }^{28}$. Nous ne sommes pas sans savoir par ailleurs que l'autonomie, qu'elle soit examinée à travers le prisme de l'éthique, du droit, de la psychologie, de la sociologie ou d'autres disciplines, demeure très complexe, difficile à mesurer, polysémique, voire tyrannique, hégémonique, pervertie! Son respect pose de réels dilemmes ${ }^{29}$. Le patient psychiatrique peut à l'évidence être un individu dangereux et nécessiter des mesures visant à le protéger ou à nous protéger. Là, soyons honnête, l'exercice de son droit à l'autonomie doit être véritablement contraint: "quelle violence à l'égard du patient et quelle violence à l'égard du soignant sont tolérables ? Où est la limite ? $^{30} »$.

Et «le bien»? La notion de bien est devenue problématique. Le professeur Fuchs a même récemment intitulé un de ses ouvrages : Comment faire pour bien faire ?31. Oui, c'est vrai, comment prendre une décision juste? Le normal, le juste, le légitime sont plus que jamais incertains. Sachant tout cela, nous devons quitter l'aparté pour revenir à notre point de vue critique sur l'enfermement du fou.

Un consentement à la fois libre et éclairé. Libre, dans la mesure où il est exempt de menaces, de manipulations ou de toute autre forme de pressions, de peur des représailles ou d'abandon, de "coercition subtile, de la conviction dont le médecin peut témoigner, de ses propres tendances thérapeutiques personnelles conservatrices ou, au contraire, agressives ${ }^{32} »$. Dans quelle mesure sommes-nous réellement libres de consentir dans le cadre de la relation soignant-soigné ? Fort heureusement, nous ne sommes pas les premiers et les seuls à nous en inquiéter.

Éclairé, dans la mesure où il comporte un certain nombre d'informations nécessaires à la prise de décision, soit « la nature et l'objet du traitement proposé, les bénéfices escomptés, les risques prévisibles et probables qui lui sont associés, les autres alternatives possibles et disponibles et, enfin, les conséquences d'un refus de traitement».

Nous admettons néanmoins qu'il existe de véritables balises établies, soit par les lois, soit par les tribunaux, pour contrôler l'exercice du pouvoir psychiatrique sur le fou et le protéger. C'est vrai que l'esprit des différentes lois applicables et l'interprétation qu'en font nos tribunaux sont animés de la volonté de protéger les personnes de façon générale.

À titre d'exemple, pour atteindre ces objectifs la Loi sur la protection des personnes dont l'état mental présente un danger pour elles-mêmes ou pour autrui prévoit un certain nombre de règles substantives et procédurales devant être respectées par les établissements de même que l'existence de recours pouvant être exercés par ces personnes ${ }^{33}$. Par exemple, la garde forcée d'une personne, dans la mesure où elle suppose de toute évidence une privation de sa liberté et sa détention, contre son gré, dans un établissement, porte gravement atteinte aux droits garantis notamment par les chartes des droits et libertés. Aussi, toutes les dispositions législatives devront être interprétées de façon restrictive et appliquées avec le souci de respecter néanmoins les droits fondamentaux de la personne. On pense au droit à l'information concernant les motifs de la garde, les droits et les recours qu'elle possède et la date de la fin de la garde. Au chapitre des droits de la personne sous garde, on retrouve également les droits à la communication avec les proches, avec un avocat, le droit d'être transféré dans un autre établissement, etc.

Il y a eu également un certain nombre de décisions judiciaires fort controversées du reste, et dont nous avons déjà fait l'analyse par le passé, qui, au moment d'accorder l'autorisation judiciaire de traitement contre le gré d'une personne, ordonnaient au médecin, peut-être maladroitement, de transmettre un rapport au comité d'éthique de l'établissement afin d'être assuré que le traitement du patient demeure conforme aux règles de l'éthique ${ }^{34}$. Au centre des préoccupations de ces juges: l'intérêt du patient. Il s'agissait en effet de voir à ce que l'autorisation du tribunal ne consiste pas en un simple blanc-seing donné au médecin traitant.

\section{QUAND LE FOU}

\section{S'EN VA-T-EN GUERRE...}

On l'a vu, l'aptitude du fou à décider de son propre bien a été reconnue par le droit. Nous voilà maintenant en présence d'un fou qui a décidé que le traitement que lui proposent les soignants n'est pas dans son meilleur intérêt. Il refuse. Nous devons aller en procès puisque la loi l'impose. En effet, dans la mesure où l'on ne peut administrer quelque traitement que ce soit à un patient apte contre son gré, il faudra aux soignants convaincus que le traitement doit néanmoins être appliqué présenter une requête devant le tribunal: la Requête pour autorisation de traitement ou la Requête pour évaluation psychiatrique ou la Requête pour mise sous garde en établissement à la suite d'une évaluation psychiatrique ou, encore, celle pour renouveler cette ordonnance.

Or, on verra dans les faits que «tout ce qui brille n'est pas or ». Souvenons-nous que les acteurs du droit et de la psychiatrie débattent dans un espace, un temps et des pratiques ritualisées qu'on appelle le procès, constitué pour Bourdieu de rapports de domination et qui représente « une mise en scène paradigmatique de la lutte symbolique dont le monde social est le lieu ${ }^{35} »$.

Nous envisagerons trois situations.

\section{Le procès a lieu;}

le fou en est-il informé, est-il présent?

Quasi absents ou même, dans certains cas, totalement absents de l'action, du dénouement d'une histoire, la leur, pourtant au cœur de leur destinée, les fous n'ont pas tort de penser que la mise en œuvre de leur droit à l'autodétermination est le plus souvent exposée à des risques majeurs tant il est vrai que les acteurs professionnels du procès restent maîtres de ce champ de bataille. Une tragique méprise.

Nous en voulons pour preuve l'existence et l'utilisation qu'on pourrait juger abusive, ou en tout cas contestable, de procédures judiciaires visant à les exclure du prétoire, du lieu du débat concernant justement le traitement qu'ils ont refusé et qu'on voudrait leur administrer contre leur gré et pour leur bien. Nous ne pouvons résister à l'envie de prendre, comme Antisthène et Diogène au fond de leur solitude, une attitude cynique en posant cette question: a-t-on déjà vu les tribunaux être saisis d'une affaire concernant un traitement auquel un patient aurait consenti?! C'est peutêtre à l'occasion du refus, dont se sentent victimes les professionnels de la santé, que les systèmes du droit et de la clinique font le mieux alliance.

Examinons ces différentes questions sous l'angle de l'effectivité du droit, c'est-àdire en observant le degré de réalisation, dans les pratiques sociales, des règles énoncées par le droit. Baissons le masque des lois quelques instants pour voir de quelle façon elles sont appliquées dans les faits. Là, dans le jargon juridique, mettre un nom sur le double visage de la justice c'est dire «dispense », un savoureux mélange de bienfaits, de confiance et d'autorisation exceptionnelle accordée en vue de protéger notre ami le fou, celui-là même dont on dit qu'il déraisonne. D'abord, la «dispense de signification » qui prévoit, à l'article $779 \mathrm{du}$ Code de procédure civile:

La demande ne peut être présentée au tribunal ou au juge à moins d'avoir été signifiée à la personne qui refuse l'évaluation ou la garde au moins un jour franc avant sa présentation. 
Cette demande est aussi signifiée à une personne raisonnable de sa famille ou, le cas échéant, au titulaire de l'autorité parentale, au tuteur, curateur, mandataire ou à la personne qui en a la garde ou qui démontre un intérêt particulier à son égard ; à défaut, la demande est signifiée au curateur public.

Exceptionnellement, le juge peut dispenser le requérant de signifier la demande à la personne concernée s'il considère que cela serait nuisible à la santé ou à la sécurité de cette personne ou d'autrui, ou s'il y a urgence.

Puis, la « dispense d'interrogatoire », à l'article 780 du même Code :

Le tribunal ou le juge est tenu d'interroger la personne concernée par la demande, à moins qu'elle ne soit introuvable ou en fuite ou qu'il ne soit manifestement inutile d'exiger son témoignage en raison de son état de santé ; cette règle reçoit aussi exception lorsque s'agissant d'une demande pour faire subir une évaluation psychiatrique, il est démontré qu'il y a urgence ou qu'il pourrait être nuisible à la santé ou à la sécurité de la personne concernée ou d'autrui d'exiger le témoignage.

Dans les deux cas, on constate que la dispense peut être accordée au motif que le fait de connaître l'existence d'une telle requête ou d'être interrogé par le tribunal "pourrait être nuisible à la santé ou à la sécurité du fou ou d'autrui, ou s'il y a urgence ». Qu'est-ce à dire? Il s'agit bien sûr d'exceptions, le principe étant qu'on signifie et/ou qu'on interroge.

Par ailleurs, si l'on consulte le fameux $\operatorname{Kelada}^{36}$, véritable mode d'emploi de la rédaction des procédures pour les praticiens du droit, on lit dans les «modèles» relatifs à la Requête pour évaluation psychiatrique et à la Requête pour mise sous garde en établissement à la suite d'une évaluation psychiatrique ou encore pour renouveler cette ordonnance:

Compte tenu qu'il serait nuisible

à la santé (et/ou à la sécurité) de

l'intimé (et/ou d'autrui) de lui signifier

la présente requête (ou Vu l'urgence),

le requérant demande d'être dispensé

de signifier la présente requête

à l'intimé ;

Compte tenu qu'il serait nuisible

à la santé (et/ou à la sécurité)

de l'intimé (et/ou d'autrui) d'exiger

son témoignage (ou Vu l'urgence),

le requérant demande de dispenser

l'intimé de témoigner ;

Dans les circonstances, il est

manifestement inutile d'exiger

le témoignage de l'intimé, en raison

de son état de santé.
Est-ce à dire que la demande de ces dispenses se fait de manière quasi automatique, s'agissant justement de modèles? Faut-il suivre le modèle? Le Petit Robert définit le modèle comme étant «ce qui sert ou doit servir d'objet d'imitation pour faire ou reproduire quelque chose».

Mais qu'en est-il de la fréquence à laquelle on recourt à ces dispenses? Fréquence - dispense: déjà deux rimes riches pour notre oreille; imaginons un peu comment elles s'accordent entre plaideurs, psychiatres et juges! Notre aptitude à éprouver l'art poétique s'arrête là. À regret, on doit constater que l'existence d'un débat contradictoire est peut-être menacée quand il s'agit d'un fou. C'est une douche froide, très froide, d'une lassitude indicible pour ceux et celles qui se battent pour que les lois visant à protéger les droits humains ne jouent pas seulement pour les banquettes pendant que celles qui visent à protéger les pouvoirs format géant, toutes catégories confondues, continuent de tenir les premières.

\section{Le procès a bel et bien lieu, le fou est présent, l'entendrons-nous?}

Dans un deuxième temps de la démarche, qu'arrive-t-il si la dispense de signifier la requête au fou n'est pas accordée? Il peut alors témoigner. Or, qu'a-t-on dit de lui avant qu'il ne fasse le serment de dire toute la vérité ? En d'autres mots, qu'est-ce que le juge a déjà entendu, de qui ? Comment entendra-t-il le fou qu'il interroge selon les normes usuelles qu'il pratique?

Il a entendu un avocat, le plus souvent expérimenté en matière de refus de consentir, assisté des experts, pour le compte de l'hôpital.

Il a entendu l'opinion d'un expert. Une « expertise».

Il interroge un fou.

Un fou répond à ses questions concernant les raisons pour lesquelles il refuse l'acte destiné à soigner sa folie.

Le fou est le plus souvent seul, sans la présence d'un avocat et d'un expert.

Il ne connaît pas les règles du jeu auquel la loi l'a convié... pour le protéger.

Lequel de ces protagonistes a le plus de plomb dans l'aile selon vous?

Ô rage! Ô désespoir! Quand le paternalisme juridique s'unit au paternalisme médical, sous couvert de protection, ils peuvent former un ménage solide d'où un débat réellement contradictoire aurait du mal à émerger : le système familial est peutêtre lui-même malade. En tout cas, lorsque la décision tombe, le patient qui se voit contraint à l'enfermement ou à la prise de substances qu'il ne veut pas absorber doit certainement se sentir d'une certaine manière tyrannisé. La loi aura-t-elle par ailleurs réussi à sauver les apparences de sa liberté ?
Qu'il est difficile d'abandonner le préjugé qu'une personne qui subit un diagnostic psychiatrique est nécessairement dépourvue de la capacité de consentir ou de refuser un traitement; de la faculté, par conséquent, d'exercer ses droits à l'autonomie, à l'intégrité, à l'inviolabilité, à l'égalité et à la dignité, pourtant des droits fondamentaux consentis à toute personne.

Pour Gendreau, le jugement que l'on pose sur la capacité de consentir ou de refuser un traitement quand il s'agit d'un patient psychiatrique est justement la pierre angulaire du problème car il forme le lieu obligé de la prise en compte effective de sa parole. Admettons en effet que si le droit de consentir ou de refuser ne lui est pas réellement reconnu, le furiosos est réduit à une fureur sans parole. Salas en fait l'enjeu philosophique de toute cette question:

Ni plus ni moins que de reconnaître le sujet atteint d'un trouble mental comme sujet de droit quel que soit son état psychologique. Non pas, sans doute, un sujet ayant pleine capacité juridique mais, à tout le moins, un sujet de droit-créance et surtout un sujet de parole ${ }^{37}$.

Les patients et leurs familles sont également des acteurs de cette œuvre dramatique, mais ils y seraient pour l'essentiel réduits au rôle de simples figurants. Il n'est d'ailleurs pas banal de noter que, suivant l'usage commun, ce type de personnage est précisément défini comme étant effacé, quand il n'est pas carrément muet:

La négociation, dont le destin social du malade est le résultat, n'a pas lieu

entre l'expert et ceux qui posent

le problème, mais entre l'expert

et d'autres experts ou d'autres

responsables qui ont mandat

(et pouvoir) de résoudre le problème.

C'est toujours une question d'équilibre, d'échanges, de concurrence entre représentants d'appareils: de la justice, de l'administration, de la police...

S'il existe quelqu'un à qui l'on a jamais demandé son avis sur son traitement, c'est bien le fou ${ }^{38}$.

$\mathrm{Au}$ procès donc, est-il réellement permis au fou d'argumenter? Est-il représenté par un avocat susceptible de prendre véritablement sa défense? Peut-il expliquer et faire entendre les motifs de son refus de traitement, puisque c'est à l'occasion du refus, comme on l'a vu, que l'affaire devient particulièrement problématique? Peut-il faire valoir son point de vue sur «sa » santé, sur la façon dont «il » entend la gérer, sur «sa » destinée ? A-t-il vraiment droit à la parole? Sa parole est-elle prise en considération? En principe, bien sûr. Rappelons les raisons pour lesquelles la tenue d'un débat réellement contradictoire est si importante : 
Le principe de non-contradiction enferme la pensée, au terme de l'argumentation, dans la certitude de la vérité atteinte. L'accueil de la contradiction dans le paradoxe permet de maintenir la pensée ouverte, aux prises avec l'incertitude et elle-même incertaine, par-delà ses certitudes; vivante ${ }^{39}$.

Ce qui compte ici, c'est la vie ; c'est aussi ce que disait Nothumb, citée ici en introduction, au sujet des yeux qui ont un regard...

Dans un autre ordre d'idées, comment peut-on de façon réaliste continuer d'ignorer l'existence et l'importance de variables psychiques et psychosociologiques dans les processus d'interprétation et d'application du droit? Le processus de décision est encore trop souvent pensé à travers la sacro-sainte rationalité logique du jugement. Et les affects, les intuitions, les sentiments d'équité, de justice, les émotions, les opinions? Déjà, en 1952, Gorphe défendait une telle approche psychologique du jugement :

Comme pour bien connaître autrui, il faut commencer par bien se connaître soi-même et déterminer son indice de réfraction psychologique, on peut se demander s'il ne servirait pas aux juges de se faire au moins sommairement psychanalyser... Accroître le savoir sans former la volonté ne fait pas avancer l'art du jugement ${ }^{40}$.

Enfin, un grand nombre de recherches en psychologie sociale démontrent que la rencontre avec l'étrangeté d'un phénomène, en l'occurrence la folie, le degré de proximité et l'implication des personnes présentes à cette rencontre, pourraient renforcer le pouvoir des représentations individuelles et collectives. Schurmans propose que les divergences d'opinion soient clairement exposées par les soignants:

Il conviendrait de suspendre consciemment un conflit d'interprétation s'exprimant en termes de légitimité (mon interprétation est plus légitime que la vôtre dans la mesure où je suis spécialiste) pour entrer dans une logique de plausibilité (différentes interprétations coexistent, légitimement: elles sont l'une et l'autre plausibles), dans la mesure où, effectivement, la médecine elle-même a produit des interprétations divergentes des pathologies psychiques ${ }^{41}$.

\section{Le procès a bel et bien lieu, le fou est présent,} mais le témoin-expert est aussi au rendez-vous...

Pour mieux appréhender la réalité clinique, les principes scientifiques recon- nus dans le traitement des différentes pathologies psychiatriques et les meilleures pratiques de soins, on aura besoin d'un interlocuteur privilégié: le témoin-expert. Les pairs seraient généralement les mieux placés pour déterminer ce qui doit ou ne doit pas être fait. Ils sont représentés devant le tribunal par les expertises et les références scientifiques et servent à « éclairer » le juge quant à la meilleure décision à prendre. Or, en l'occurrence, il faut savoir que le pouvoir psychiatrique et l'autonomie professionnelle de ses agents, les psychiatres, sont encore plus forts que dans le cas des autres disciplines médicales en plus d'être légitimés par un consensus social et politique sans pareil ${ }^{42}$.

Qui est l'expert? Ici, souvenons-nous, c'est celui à qui le fou a dit non. C'est celui dont le fou a refusé les bons soins. Le refus de soins ou de traitements s'avère un reflet insoutenable pour un professionnel de la santé de ce qu'il est sur un plan professionnel. Une sacrée égratignure narcissique, un rejet, une non-reconnaissance de ce que lui, l'expert, est en mesure d'apporter, de ce pourquoi il a été formé et de ce pourquoi il a choisi d'être formé dans ce champ, de sa compétence professionnelle, de l'intention presque naturelle qu'il a de faire le bien. Un «reflet» est une image réfléchie, généralement atténuée certes, mais tout de même un écho, une imitation, une représentation. Or, comment s'éprendre de soi en se regardant dans l'eau d'une fontaine à travers un reflet aussi peu éclatant? En fait, les soignants souffrent de ces situations, plus sérieusement.

Mais il n'en demeure pas moins que le miroir et son reflet peuvent être des outils féconds pour saisir en quoi les comportements individuels et collectifs d'exclusion et de pathologisation de la folie - surtout lorsque les fous refusent d'être soignés peuvent également être porteurs d'un imaginaire marqué au coin de la peur, des peurs et des certitudes, celles-là, par contre, liées immanquablement au pouvoir ${ }^{43}$.

Face au refus, plusieurs attitudes sont possibles. Ainsi, le professionnel peut afficher une attitude autoritaire en choisissant d'imposer le traitement. Le malade sera alors puni pour refus d'obéissance, pour refus de se soumettre, "car la désobéissance équivaut à une déclaration de guerre contre votre personne. Votre fils veut vous prendre le pouvoir et vous êtes en droit de combattre la force par la force, pour raffermir votre autorité ${ }^{44}$ ». Admettons tout de même que l'impuissance à laquelle le respect de l'autonomie condamne les soignants à l'heure actuelle, qui s'accompagne pour certains auteurs d'une certaine crise de l'autorité professionnelle et d'une dévalorisation de l'expertise, aurait de quoi susciter de la colère et dérouter, pour dire le moins ${ }^{45}$. L'autonomie et son pendant, l'exigence du consentement, sont devenues de véritables contre-pouvoirs. Mais continuons.

Le droit intervient au moment où le professionnel adopte la première attitude. La règle de droit prévoit en effet qu'on ne peut administrer un traitement à quiconque contre son gré - même un fou - à moins d'avoir obtenu l'autorisation du tribunal. Le psychiatre peut-être autoritaire doit donc aller défendre sa position devant le juge pour pouvoir aller de l'avant.

Par ailleurs, cet expert est-il neutre, objectif? De plus, qui est-il et que communiquera-t-il aux autres acteurs professionnels du procès? Est-il le représentant de l'idéologie dominante du groupe professionnel auquel il appartient, de la société à laquelle il appartient? Comment cet échange d'informations et de valeurs influence-t-il le jugement qu'il s'agit de poser $^{46}$ ?

On sait que lorsque s'affrontent deux ou plusieurs écoles de pensée qui jouissent de fondements sérieux et également défendables, le tribunal n'interviendra pas. Cependant, s'il y a simple divergence au niveau de l'opinion des experts, le juge qui entend la cause pourra choisir l'opinion la plus probable, la plus conforme à la preuve, la plus crédible. Mais encore faut-il que ces opinions divergentes soient présentes au procès. Le sont-elles si le fou n'y est pas représenté par un avocat ou s'il est seul?

Par ailleurs, le droit, discours de pouvoirs, est pourtant vulnérable - eh oui - face à l'autonomie professionnelle, face à l'expert, cet «être au-dessus de tout soupçon », comme le qualifie Bozzini ${ }^{47}$. Entre ombres et lumières : l'ordre juridique possède également ses zones de fragilité. $\mathrm{La}$ définition même du professionnel servirait à justifier et à maintenir le dogme de l'autonomie professionnelle et, avec lui, le pouvoir. Le mot est de nouveau lancé:

Les abus qui pourront découler de

l'exercice de ce pouvoir se manifestent notamment jusque dans le pouvoir de mesurer et de définir les besoins des citoyens et la manière de les satisfaire.

Un pouvoir qui fait mal, dans

le contexte de la santé,

à l'action autonome du patient ${ }^{48}$.

En fait la définition de ce qu'est un professionnel suggère l'image de deux personnes, l'une, compétente et l'autre, ignorante. Elle contient virtuellement la violence de n'être pas reconnu comme un sujet compétent, en tant que personne soignée, et par voie de conséquence, comme un sujet apte à déterminer ce que constitue son propre bien $^{49}$. Alors, l'autre est une menace. Nous nous trouvons face à une crise indicible de la confiance. Sur le plan éthique, face à 
l'exigence d'informer le patient, on distingue trois modèles d'attitudes: paternaliste, informatif ou délibératif ${ }^{50}$. Le modèle délibératif postule que le soignant et le soigné possèdent des compétences, différentes certes, mais bel et bien existantes au sein de la relation. Fondé sur la négociation entre deux partenaires compétents, il insiste sur le fait que la recherche du consentement de la personne repose sur un processus, un dialogue et des aptitudes relationnelles respectueuses de la personne soignée, surtout lorsqu'il s'agit de personnes particulièrement vulnérables. Ici, le comment faire, le comment dire sont aussi importants que le quoi dire.

Le processus judiciaire que nous avons décrit et remis en cause, le verbe sans aucun doute cinglant voire impertinent, respectet-il ces nouvelles exigences éthiques et humanistes? La sollicitude, la compassion, la justice, la responsabilité, en tant que condition de notre humanité, sont-elles véritablement au rendez-vous de la rencontre $\mathrm{du}$ fou avec les acteurs du droit et de la clinique et ce, tout au long du processus, difficile par ailleurs? Pourrait-on même aller jusqu'à se reconnaître dans le fou et lui reconnaître, comme le disent Blondeau et Gagnon, une certaine grandeur, dans la mesure où «il représente celui qui refuse l'aliénation à la norme et la négation de sa singularité uniquement pour se conformer aux attentes des autres. Le délire est souvent une manière de se faire entendre, disait Freud, une construction en réponse à un mal, et non le mal lui-même ${ }^{51} »$ ? Si la folie «contenait une vérité ${ }^{22} » ?$

Peut-on aussi réellement parler d'autonomie quand l'expression du «je», par le fou, est à ce point à risque d'être enfermée dans le regard de l'autre? Peut-on aussi parler d'autonomie, pour nous, quand notre propre expression de professionnel est prisonnière de nos préconceptions? Le filtre folie-maladie, à travers lequel on regarde le fou, ne porte-t-il pas en lui-même la mort de l'esprit du fou en même temps que la nôtre?

L'épanouissement de la vie de l'esprit est-il envisageable dans l'enfermement de la pensée? Sommes-nous capables d'intégrer le doute, la mise à distance, dans nos processus de compréhension et de prise de décision? Savons-nous, êtres bienpensants, douter de nous, peut-être même dans certains cas nous méfier de nousmêmes, de nos jugements, mais surtout de leurs fondements? L'avons-nous seulement appris? Pouvons-nous l'apprendre?

Qui est le fou?

Entre raison et déraison. Était-il si impertinent de poser la question?

\section{Notes}

1 R. HOUDE, Les temps de la vie. Le développement psychosocial de l'adulte selon la perspective du cycle de vie, Montréal, Gaëtan Morin, 1999, p. 246.

2 A. NOTHUMB, La métaphysique des tubes, Paris, Albin Michel, 2000, p. 7-9.

3 Avant de poursuivre cette réflexion, nous ressentons le besoin d'aviser le lecteur que nous avons choisi d'opter pour une explication qui, à sa façon, "implique le mal», selon l'expression savoureuse de J. CARBONNIER, Flexible droit. Pour une sociologie du droit sans rigueur, Paris, LGDJ, 1988, p. 124: "Réfléchissons-nous assez à tout ce que le pessimisme de Voltaire contenait de possibilités méthodologiques? Entre deux explications pareillement acceptables d'un phénomène, il serait sans doute de bonne méthode d'opter pour celle qui implique le mal [...] En sociologie du droit, ce pourrait être un instrument d'analyse affreusement efficace. »

4 G. BOURGEAULT, Éloge de l'incertitude, Montréal, Bellarmin, 1999, p.44.

5 E. JABÈS, Le livre des questions, Paris, Gallimard, 1963.

6 C. BOBIN, La folle allure, Paris, Gallimard, 1995, p. 49. Il convient cependant de souligner qu'avec le remplacement de la Loi sur la protection du malade mental par la Loi sur la protection des personnes dont l'état mental présente un danger pour ellesmêmes ou pour autrui, la terminologie utilisée pour désigner cette personne a ellemême peut-être opéré un renversement du regard. On ne parle plus de malade mental comme c'était le cas autrefois. Dorénavant, l'état mental et le caractère dangereux d'une personne constituent les critères de base de l'application de la loi, peu importe qu'un diagnostic ait été ou non posé.

7 M.-N. SCHURMANS, Représentations et action. Déterminants psychosociaux des politiques de soins, 1999, p. 16, texte inédit.

8 R. LETENDRE, D. MONAST et F. PICOTTE, Dynamique de l'expérience de l'hospitalisation en département interne de psychiatrie chez les 18-30 ans, Montréal, Département de psychologie, UQAM, 1988, p. 590-591 et dans C. GENDREAU, Le droit du patient psychiatrique de consentir à un traitement: élaboration d'une norme internationale, Montréal, Thémis, 1996, p. 3. On verra G. PETRILLO (dir. publ.), La santé et la maladie comme phénomènes sociaux, Paris, Delachaux \& Niestlé, 2000.

9 M. FOUCAULT, Histoire de la folie à l'âge classique, Paris, Gallimard, 1972. L'expression est de D. SALAS, "L'homme qui chavire ", dans Justice et psychiatrie: normes, responsabilité, éthique, dans C. LOUZOUN et D. SALAS (dir. publ.), Ramonville St-Agne, Erès, 1998, p. 305. On verra, généralement, "Éthique, morale et loi en psychiatrie", Cahiers psychiatriques, vol. 27, 1999 ; "L'éthique en psychiatrie et le consentement aux soins», Soins Psychiatrie, vol. 161, 1992 ; "Folies / Espaces de sens", Anthropologie et Société, vol. 1-2, 1993, dont E. CORIN, "Les détours de la raison.
Repères sémiologiques pour une anthropologie de la folie »; H. GRIVOIS, Le fou et le mouvement $d u$ monde, Paris, Grasset, 1995 ; A. FAGOT-LARGEAULT, "Éthique du consentement en psychiatrie», dans C. LOUZOUN et D. SALAS (dir. publ.), Justice et psychiatrie: normes, responsabilité, éthique, ibid., p. 77 et suiv.; S. RAMEIX, «Du paternalisme des soignants à l'autonomie des patients? ", dans C. LOUZOUN et D. SALAS (dir. publ.), Justice et psychiatrie: normes, responsabilité, éthique, ibid., p. 65 ; R. McCLELLAND et G. SZMUKLER, "Consent and Capacity in Psychiatric Practice Revisited », Eur. J. Health L., vol. 7, 2000, p. 47 ; MINARD, Folie et psychiatrie, Ramonville St-Agne, Erès, 1997.

10 I. LASVERGNAS-GREMY, "Angoisse dans l'air du temps ", Frontières-Peur bleue, vol. 12, 2000, p. 44.

11 F. CHAUMON, «L'excès de l'acte", Société, vol. 17, 1997, p. 53; G. SWAIN, Dialogue avec l'insensé, Paris, Gallimard, 1994.

12 H. ARENDT, Eichmann à Jérusalem. Rapport sur la banalité du mal, Paris, Gallimard, 1966.

13 J.-F. MALHERBE, Technique et humanisme. Une alliance contre nature?, Sherbrooke, GGC, 2000, p. 35.

14 M.-N. SCHURMANS, Représentations et action. Déterminants psychosociaux des politiques de soins, ibid., note 7, p. 2; E. DURKHEIM, "Représentations individuelles et représentations collectives ", Revue de métaphysique et de morale, t. VI, 1998 ; S. MOSCOVICI, La psychanalyse, son image, son public, Paris, PUF, 1976.

15 Ibid., p. 7-8. Aussi id., Maladie mentale et sens commun, Neuchâtel-Paris, Delachaux \& Niestlé, 1990 ; id., "Maladie mentale: fait de nature, concept et représentation sociale», Journal de psychiatrie, vol. 4, 1989; id., "Maladie mentale et altérité: deux thêmata antagonistes », dans G. PETRILLO (dir. publ.), La santé et la maladie comme phénomènes sociaux, Paris, Delachaux \& Niestlé, 2000.

16 C. GENDREAU, ibid., note 8, p. 12; D. WEISSTUB, «Le droit et la psychiatrie dans leur problématique commune", R.D. McGill, vol. 30, 1985, p. 221.

17 «Par valeur, nous référons à la notion d'idéal, la valeur étant: une manière d'être ou d'agir qu'une personne ou une collectivité reconnaissent comme idéales et qui rend désirables ou estimables les êtres ou les conduites auxquels elle est attribuée », dans C. GENDREAU, ibid., note 8, p. 60, qui cite G. ROCHER, Introduction à la sociologie générale, Paris, HMH, 1968, p. 72.

18 «L'altération des facultés mentales de certains malades les expose tout particulièrement au manque d'attention et ne leur permet pas de profiter des services de santé et de protection sociale alors même qu'ils sont prévus pour eux ", dans C. GENDREAU, ibid., note 8, p. 159.

19 Ibid., p. 12-13.

20 Ibid., p. 161-162.

21 Ibid., p. 163-164. 
22 Ibid., p. 164 à 169, pour toute cette section ; L. GOSTIN, «Consent to unusually hasardous, unestablished or irreversible treatments in psychiatry: a review of the Draft United Nations Guidelines for the protection of persons suffering from mental health », dans ASSOCIATION INTERNATIONALE DE DROIT PÉNAL, The Protection of the Persons Suffering from Mental Disorder, Nouvelles études pénales, Toulouse, Erès, 1980 ; E. GAGNON, "L'avènement médical du sujet. Les avatars de l'autonomie en santé », Sciences sociales et santé, vol. 16, 1998, p. 49-74.

23 Même le diagnostic de maladie mentale poserait un problème: W. REICH, «Psychiatric Diagnosis as an Ethical Problem» et W. FULFORD, «The Concept of Disease», dans S. BLOCH, P. CHODOFF, Psychiatric Ethics, Oxford Medical Publications, 1999, p. 77 à 134.

24 Pour l'ensemble de cette section: R. P. KOURI, S. P. NOOTENS, «Le corps humain, l'inviolabilité de la personne et le consentement aux soins", Sherbrooke, Revue de droit de l'Université de Sherbrooke, 1999, p. 213 à 273 ; A. NOLET, «Gardé contre mon gré», Justice santé, vol. 1, 2001 ; E. DELEURY et D. GOUBAU, Le droit des personnes physiques, Cowansville, Yvon Blais, 1997; Développements récents en droit de la santé mentale, Cowansville, Yvon Blais, 1998; F. VILLENEUVE, «Quand l'état mental d'une personne devient dangereux. Connaissez-vous la nouvelle loi ? ", L'Infirmière du Québec, vol. 6, 1999, p. 19 et suiv.; K. GLASS, «Refining Definitions and Devising Instruments: Two Decades of Assessing Mental Competence», Int. J. of Law and Psychiatry, vol. 20, 1997, p. 5 ; B. DICKENS, «Legal Aspects of the Dementias », The Lancet, vol. 349, 1997, p. 948 ; D. BLONDEAU et E. GAGNON, «De l'aptitude à consentir à un traitement ou à le refuser: une analyse critique ", Les Cahiers de droit, vol. 35, 1994, p. 651; Ibid., "Consentement aux soins et inaptitude», dans D. BLONDEAU (dir. publ.), Éthique et soins infirmiers, Montréal, PUM, 1999, 177-189; K. BROWN, E. MURPHY, «Falling Through the Cracks: The Quebec Mental Health System », McGill Law Journal, vol. 45, 2000, p. 1037.

25 (1991) R.J.Q. 1969 (C.S.), p. 1973.

26 J. ARBOLEDA-FLORENZ, «Le consentement en psychiatrie: la position de l'Association des psychiatres du Canada », Revue canadienne de psychiatrie, vol. 36, 1988, p. 321.

27 Centre hospitalier de la Mauricie c. C., (1998) R.L. 383, à la page 389.

28 Se pose ici la question de l'objet de ce consentement. En principe, le droit du patient psychiatrique de consentir vaut pour tous les traitements médicaux. Toutefois, comme l'observe C. Gendreau: «les catégories mêmes de traitements psychiatriques suscitent la controverse et en font un enjeu spécifique $\mathrm{du}$ droit au consentement », dans Le droit du patient psychiatrique de consentir à un traitement: élaboration d'une norme internationale, ibid., note 8, p. 36 à 43 .
29 E. FUCHS et al., «La notion d'autonomie: une reformulation interdisciplinaire», Cahiers médico-sociaux, vol. 41, 1997, 161-180; S. RAMEIX, «Du paternalisme des soignants à l'autonomie des patients? ", dans Justice et psychiatrie, ibid., note 9.

30 D. BLONDEAU, E. GAGNON, «Consentement aux soins et inaptitude », ibid., note 24, p. 183.

31 E. FUCHS, Comment faire pour bien faire?, Genève, Labor \& Fides, 1996, 196 p.

32 Lisons R. P. KOURI, S. P. NOOTENS, «Le corps humain, l'inviolabilité de la personne et le consentement aux soins », ibid., note 24 , p. 226: «La liberté du consentement réfère à une décision prise en dehors de toute influence ou contrainte indue: seule s'exprime la volonté de la personne, souveraine dans l'appréciation de son meilleur intérêt. Cette notion est, à l'évidence, toute relative: chaque individu se trouve dans un réseau complexe d'influences humaines, matérielles, environnementales, qui vont interférer avec son libre arbitre, à tel point que, pour certains, le consentement libre en matière médicale est un leurre. »

33 A.-M. VEILLEUX et H. ALLARD, « Les recours et la représentation du patient psychiatrique selon la nouvelle Loi sur la protection des personnes dont l'état mental présente un danger pour elles-mêmes ou pour autrui», dans Développements récents en santé mentale, ibid., note 24, p. 147 et suiv.

34 M.-I. FOREST, «Le comité d'éthique d'un établissement de santé ou de services sociaux est-il garant de la protection des droits de la personne?", Revue juridique Thémis, vol. 28, 1994, p. 263 et suiv.

35 P. BOURDIEU, «La force du droit; pour une sociologie du champ juridique », dans Actes de la Recherche en Sciences Sociales, no 64, Paris, 1986, p. 12.

$36 \mathrm{H}$. KELADA, F. PAYETTE, Formulaire de procédure civile, Montréal, Wilson \& Lafleur, 2000. Me Payette précise dans son avant-propos: "L'expérience l'a démontré : le Formulaire répond indéniablement aux attentes de la communauté juridique auprès de qui il a toujours joui d'un accueil très favorable. Le Formulaire a été conçu comme un guide utile et pratique qui réunit les éléments essentiels devant se retrouver dans les allégations et conclusions d'une procédure. »

37 D. SALAS, «L'homme qui chavire», ibid., note 9 , p. 307.

38 R. CASTEL, L'ordre psychiatrique, Paris, Minuit, 1976, p. 155.

39 G. BOURGEAULT, Éloge de l'incertitude, ibid., note 4, p. 48.

40 F. GORPHE, La décision de justice, étude psychologique et judiciaire, Paris, Sirey, 1952; Id., La critique du témoignage, Paris, 1927 ; Id., "La psychologie appliquée en justice», dans Traité de psychologie appliquée, Paris, PUF, 1959. On verra, en français, PAIN et al., "L'approche systémique », dans Revue de pratique de l'institutionnel, no 4, 1987 ; A.-J. ARNAUD et al. (dir. publ.), Dictionnaire encyclopédique de théorie et de sociologie du droit, Paris, LGDJ, 1988, p. 487 et suiv.

41 Représentations et action. Déterminants psychosociaux des politiques de soins, ibid., note 7, p. 15; G. MUGNY et F. CARUGATI, L'intelligence au pluriel, Cousset $(\mathrm{CH})$, DelVal, 1985.

42 E. FREIDSON, La profession médicale, Paris, Payot, 1984.

43 Dans son Éloge de l'incertitude, ibid., note 4, p. 45, Bourgeault rappelle que: «La certitude a partie liée avec le pouvoir, concrètement avec les pouvoirs [...]. J'utilise le pluriel pour rendre évidente la contradiction: l'absolu, qu'il soit vérité ou pouvoir, exige l'exclusive. S'arrogeant la totale possession de la vérité ou du pouvoir, peu importe, puisqu'il s'agit toujours d'une «vérité » mise au service d'un pouvoir et que celui-ci, en retour, protège et défend - il se fait totalitaire. Nécessairement. »

44 A. MILLER, C'est pour ton bien. Racines de la violence dans l'éducation de l'enfant, Paris, Aubier, 1983, p. 1.

45 D. BLONDEAU, E. GAGNON, «Consentement aux soins et inaptitude», ibid., note 24 , p. $178,185$.

46 M.-I. FOREST, «Jalons d'une réflexion critique sur la revendication de l'éthique en contexte clinique», dans G. GIROUX (dir. publ.), La pratique sociale de l'éthique, Montréal, Bellarmin, 143-198, aux pages 179-182; Id., «Le mythe de l'objectivité d'un jugement sur la douleur: le droit et la clinique nez à nez?", Frontières - La souffrance en question, vol. 8, no 2, 1995, p. 24-31.

47 L. BOZZINI, «L'expertise et la hiérarchie sanitaires en question », dans L. BOZZINI et al. (dir. publ.), Médecine et société. Les années 80, Montréal, St-Martin, 1986, p. 393.

48 Ibid., p. 394. En 1977, I. ILLICH, Disabling Professions, Londres, Boyars, 1977, allait plus loin en écrivant «[...] les nouvelles professions dominantes exigent le contrôle des besoins humains, tout court ». On verra B. KOUCHNER, La dictature médicale, Paris, Laffont, 1996.

49 G. LEBEER, «La violence thérapeutique », Sciences sociales et santé, vol. 15, 1997, 69-96, sur l'interprétation psychologique des conduites de résistance des patients.

$50 \mathrm{G}$. DURAND, Introduction générale à la bioéthique. Histoire, concepts et outils, Montréal, Fidès / Cerf, 1999, p. 237 et suiv.

51 D. BLONDEAU, E. GAGNON, «Consentement aux soins et inaptitude», ibid., note 24 , p. 182.

52 Ibid. 